DIVISION OF THE HUMANITIES AND SOCIAL SCIENCES

CALIFORNIA INSTITUTE OF TECHNOLOGY

PASADENA, CALIFORNIA 91125

THE TESTABLE IMPLICATIONS OF ZERO-SUM GAMES

SangMok Lee

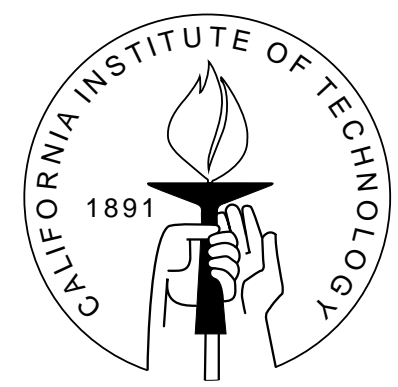

SOCIAL SCIENCE WORKING PAPER 1303

May 2009 


\title{
The Testable Implications of Zero-sum Games
}

\author{
SangMok Lee *
}

\begin{abstract}
We study Nash-rationalizable joint choice behavior under restriction on zerosum games. We show that interchangeability of choice behavior is the only additional condition which distinguishes zero-sum games from general noncooperative games with respect to testable implications. This observation implies that in some sense interchangeability is not only a necessary but also a sufficient property which differentiates zero-sum games.
\end{abstract}

\section{Introduction}

Sprumont (2000) investigates an abstract joint choice problem without assuming an explicit economic environment. He assumes that the joint behavior is simultaneous and is captured as a tuple of actions where each action is chosen by a player. Sprumont provides conditions on the testable implications such that observed joint behavior is a Nash equilibrium behavior if and only if it satisfies these conditions. They are similar to classical axioms of choice theory (see Moulin (1985)).

We retain Sprumont's basic abstract setup and ask the following question: "Is the choice function Nash-rationalizable with a certain game, specifically, zero-sum games?".

${ }^{*}$ Division of the Humanities and Social Sciences, California Institute of Technology, Pasadena, CA 91125. Email: sangmok-at-hss.caltech.edu I am grateful to Christopher Chambers and Federico Echenique for encouraging and guiding me in working on this paper. I am also thankful to Ruth Mendel and Luke Boosey for corrections and neatly reorganizing the proves. 
Zero-sum games have been studied since game theoretic research began. They are still considered important since they model a pure conflict of interest and admit a large variety of applications (for example, the resource division problem). Thus, in addition to the behavioral conditions implied by Nash-rationalizability, we may need other conditions to ensure that the joint choice function is in fact rationalized by a zero-sum game. As an introductory example, Figure 1 shows how Nash-rationalizable choice behavior may not be able to be rationalized by a zero-sum game. ${ }^{1}$
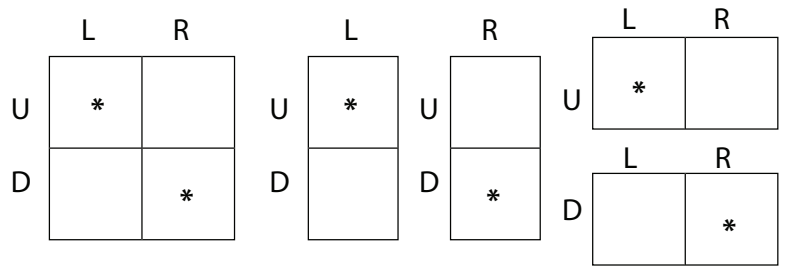

Figure 1: Nash-rationalizable but not with zero-sum games

In this example, player 1 can conceivably choose either $U$ or $D$ and player 2 may choose $L$ or $R$. However, following classical choice theory, we observe how players choose when choice sets are restricted. Figure 1 shows all the possible games from which two players may choose their joint actions. For each game, $*$ is the action profile chosen by two players. We can verify that the joint choice behavior exhibited in Figure 1 is consistent with Nash equilibrium behavior of a coordination game in which coordinating to $(U, L)$ or $(D, R)$ gives a higher payoff to both players.

However, this choice function cannot be Nash equilibrium behavior by any zero-sum game. Assuming that the choices are Nash-equilibria of a zero-sum game, we have that $(U, L)$ is chosen from $\{(U, L),(D, L)\}$ and $(D, R)$ is chosen from $\{(D, L),(D, R)\}$. These imply that for player $1,(U, L)$ is strictly preferred to $(D, L)$; for player $2,(D, R)$ is strictly preferred to $(D, L)$, which implies player 1 strictly prefers $(D, L)$ to $(D, R)$. On the other hand, since $(D, R)$ is chosen from $\{(D, R),(U, R)\}$ and $(U, L)$ is chosen from

\footnotetext{
${ }^{1}$ This example is originally from Sprumont (2000). In his paper, the example was provided to show how choice behavior appears to be Nash-rationalizable.
} 
$\{(U, L),(U, R)\}$, for player $1,(D, R)$ is strictly preferred to $(U, R)$; for player $2,(U, L)$ is strictly preferred to $(U, R)$, which implies player 1 strictly prefers $(U, R)$ to $(U, L)$. As a result, these cyclic preference orders imply that all possible joint actions are indeed indifferent for player 1 (and thus player 2 by the fact that the game is zero-sum). Therefore, we would expect to see all strategy profiles chosen.

This example shows that once we have two choices on the diagonals in a table of joint actions, in order for the joint choice function to be Nash equilibrium behavior with a zero-sum game, the other pairs of actions also should be chosen. When a choice behavior has the property that any pair of actions chosen, one for each player, is also jointly chosen, we say that the choice behavior is interchangeable. Our main theorem shows that this interchangeability of joint choice behavior is indeed the only additional condition that distinguishes the testable implications of zero-sum games from those of general non-cooperative games.

Since Samuelson introduced his analysis of revealed preference (Samuelson, 1938), there have been a considerable number of research studies on the testable implications of individual decision making. However, compared to the history and popularity of game theory and the research devoted to refining solution concepts, the falsifiability of game theoretic models has received scant attention. More recently, there have been several studies on the testable implications of collective decision-making such as household behavior (Chiappori, 1988) and general equilibrium model (Brown and Matzkin, 1996).

In game theoretic setup, while Sprumont (2000) deals with simultaneous joint decisionmaking, and therefore normal form games, Ray and Zhou (2001) consider the case in which all observed joint choices involve sequential movement by players. They derive conditions the choice behavior needs to satisfy in order to coincide with subgame perfect Nash equilibria under complete information. Subsequent work by Ray and Snyder (2003) has consolidated these two independent rationalizability concepts into one con- 
dition, which they call subgame-perfect consistency. For further references, we refer to Carvajal, Ray, and Snyder (2004).

The following section begins with a brief summary of Sprumont (2000) and states the additional condition for Nash-rationalization with zero-sum games. Section 3 discusses possible difficulties in extensions of the main theorem and Appendix A is devoted to the proof of the main theorem.

\section{Model and main theorem}

There are two players, 1 and 2. Let $A_{1}$ and $A_{2}$ be finite sets of actions that player 1 and 2 may conceivably choose in a game. $A:=A_{1} \times A_{2}$ is the set of all conceivably possible joint actions. Although each player has independent preference over joint actions, a certain action profile is chosen by both players. The game theoretic model captures this interdependent choice environment.

Following the classical revealed preference approach, suppose we observe choices when two players play a game $B=B_{1} \times B_{2}$ where $B_{1} \subset A_{1}$ and $B_{2} \subset A_{2}$ are the sets of all available actions for player 1 and 2. In this model, all choices that the players make in each game can be summarized as a choice function.

Definition 1 Let $\mathscr{A}=\left\{B=B_{1} \times B_{2} \mid \emptyset \neq B \subset A\right\}$ be the set of all non-empty Cartesianproduct sets included in $A$. A joint choice function $f$ assigns to each $B \in \mathscr{A} a$ nonempty set $f(B) \subset B$.

In the case where at most one player has more than one available action in $B$, we say $B$ is in a line. Depending on the player, the line is either a column or a row - the former when player 1 has choices, the latter when player 2 has choices. In addition, for 
any $B^{\prime \prime} \subset B$ and $B^{\prime \prime} \in \mathscr{A}$, we call $B^{\prime \prime}$ a subgame of $B$. For any $B, B^{\prime} \in \mathscr{A}$, define $B \vee B^{\prime}$ as the set of all possible pairs of actions from $B_{i}, B_{i}^{\prime},(i=1,2)$. That is,

$$
B \vee B^{\prime}:=\prod_{i=1,2}\left(B_{i} \cup B_{i}^{\prime}\right)
$$

As in Sprumont (2000), we assume that all players make choices simultaneously and the choice function is possibly multi-valued. In individual choice theory, a choice function from a set of alternatives is called rationalizable if there exists a binary relation, $\mathrm{R}$, such that the choice agrees with the maximal set in $\mathrm{R}$. The following definitions are the counterparts of rationalizability in game theory. Compared to abstract individual choice theory, which is based on a set of alternatives subject to a single binary relation, we have a set of alternative strategy profiles and two binary relations. We assume that the relations (say $\succeq$ ) have the following properties, and are termed weak orders.

i. Complete: For all joint choices $a, b \in A, a \succeq b$ or $b \succeq a$.

ii. Transitive: For all $a, b, c \in A$ for which $a \succeq b$ and $b \succeq c$, it follows that $a \succeq c$.

Definition 2 A joint choice function $f$ is Nash-rationalizable if there are two weak orders $\succeq_{1}, \succeq_{2}$ on $A$ such that, for each $B \in \mathscr{A}, f(B)$ coincides with the set of all Nash equilibria of the game $\left(B, \succeq_{1}, \succeq_{2}\right)$.

Sprumont (2000) introduces the following two conditions for Nash-rationalizability. These conditions are extended versions of Chernoff, expansion, and Sen's $\beta$ in individual choice theory. When the feasible actions are restricted to lines, "Persistence under Expansion" is exactly the same as expansion and "Persistence under Contraction" is exactly the same as Chernoff and Sen's $\beta$.

Definition 3 A joint choice function over $\mathcal{A}$ is 
- Persistent under Expansion (PE): For all $B, B^{\prime} \in \mathscr{A}, f(B) \cap f\left(B^{\prime}\right) \subset f\left(B \vee B^{\prime}\right)$.

- Persistent under Contraction (PC):

(PC1) : For all $B, B^{\prime} \in \mathscr{A}$ with $B^{\prime} \subset B, f(B) \cap B^{\prime} \subset f\left(B^{\prime}\right)$.

(PC2): Moreover, if $B$ is in a line, $B^{\prime} \subset B$ and $f(B) \cap B^{\prime} \neq \emptyset$ implies $f\left(B^{\prime}\right) \subset$ $f(B)$.

With these two conditions, Sprumont (2000) establishes the following theorem.

Theorem 4 A joint choice function $f$ is Nash-rationalizable if and only if it satisfies $(P E)$ and $(P C){ }^{2}$

From this model of Nash-rationalizability, we restrict the set of available rationalizing games from the set of all non-cooperative games to include only zero-sum games. Under the conditions of zero-sum games, the preferences of two players conflict in the opposite direction. Therefore, while a general non-cooperative game consists of two weak orders, we can describe zero-sum games with a single weak order.

Definition 5 Let $\succeq$ be a weak order over $A$. The game defined by $(A, \succeq, \preceq)$ is called $\boldsymbol{a}$ two-person zero-sum game, where $\preceq$ is the inverse relation of $\succeq .{ }^{3}$ We say that a joint choice function $f$ is Nash-rationalizable with a zero-sum game if there is a weak order $\succeq$ on $A$ such that for each $B \in \mathscr{A}, f(B)$ coincides with the set of all Nash equilibria of the game $(B, \succeq, \preceq)$.

As demonstrated in Example 1, not all Nash-rationalizable joint choice functions are Nash-rationalizable with zero-sum games. In the example, we need at least one additional

\footnotetext{
${ }^{2}$ In Sprumont (2000), this theorem holds for any $n$-agent joint choice.

${ }^{3}$ Let $\succeq$ be a binary relation over $A$. Inverse relation $\preceq$ is defined as, For all $a, b \in A$ for which $a \succeq b, b \preceq a$.

Later, we will use the fact that the inverse relation of a weak order is also a weak order. The proof is straightforward by definition.
} 
condition which fills the gap in the product space of two distinct choices in the diagonal of the game table. This condition requires that the joint choice function is interchangeable.

Definition 6 (Interchangeable choice function) A joint choice function $f$ over $\mathscr{A}$ is interchangeable if for all $B \in \mathscr{A}$ and all $b_{*}, b_{*}^{\prime}$ in $f(B),\left\{b_{*}\right\} \vee\left\{b_{*}^{\prime}\right\} \subset f(B)$.

Provided that agents play a zero-sum game and observed joint actions are the Nashequilibria of the corresponding subgame, the choice function is interchangeable. It is well-known that any pair of equilibrium strategies of a zero-sum game, one for each player, is an equilibrium strategy profile (see Luce and Raiffa (1957)). Our contribution is to show that interchangeable choice behavior is indeed the only additional condition which distinguishes zero-sum games from general non-cooperative games. The following theorem summarizes this result.

Theorem 7 A joint choice function defined on a set of two-agent actions is Nashrationalizble with a zero-sum game if and only if it satisfies (PE), (PC), and interchangeability.

Proof: See Appendix A.

\section{Discussion}

Sprumont only applies (PC2) for each line. Its only function is to guarantee transitivity. From this observation, Sprumont introduces "quasi Nash-rationalizability" where a choice function agrees with the set of Nash-equilibria of $\left(B,\left(\succeq_{1}, \succeq_{2}\right)\right)$ for all $B \in \mathcal{A}$, in which $\left(\succeq_{i}\right)_{i=1,2}$ are $P$-acyclic binary relations on $A$ (possibly non-transitive). ${ }^{4}$ He proves that a joint choice function is quasi Nash-rationalizable if and only if it satisfies (PE), (PC1).

\footnotetext{
${ }^{4}$ A binary relation $\succeq$ on $A$ is $P$-acyclic if and only if, For all $a, b, c \in A$ for which $a \succ b$ and $b \succ c, \neg(c \succ a)$.
} 
However, the following example shows that (PE), (PC1), and interchangeability are not enough to induce quasi Nash-rationalizability with zero-sum games.

\section{Example 85}

Figure 2 describes a choice function $f$ over all binary choices in $A$. The left figure is for adjacent actions in the table and the right figure is for other binary choices. For each column, a solid arrow implies that only one action is chosen against the other; a dashed line implies that both are chosen. For example, $\{b\}=f\left(\left\{b, a^{6}\right\}\right)$ and $\left\{a^{2}, c\right\}=f\left(\left\{a^{2}, c\right\}\right)$. For each row, the arrow describes the choice inversely: e.g. $\left\{a^{4}\right\}=f\left(\left\{a^{3}, a^{4}\right\}\right)$ and $\left\{b, a^{4}\right\}=f\left(\left\{b, a^{4}\right\}\right)$. Assuming that $f$ satisfies (PE) and (PC1), we can verify the choice values for each $2 \times 2$ subgames. ${ }^{6}$ For other subgames,

- $\left\{a^{2}\right\}=f($ column 1 and 2$),\left\{a^{4}\right\}=f($ column 2 and 3$)$, and $\left\{b, a^{4}\right\}=f($ column 1 and 3$)$.

- $\left\{d, a^{4}\right\}=f($ row 1 and 2$),\left\{a^{4}\right\}=f($ row 2 and 3$)$, and $\{c\}=f($ row 1 and 3$)$.

- $\left\{a^{4}\right\}=f(A)$.

\begin{tabular}{|c|c|c|}
\hline$a^{1}$ & $a^{2}$ & $d$ \\
\hline b & $a^{3}$ & \\
\hline$a^{6}$ & c & $a^{5}$ \\
\hline
\end{tabular}

\begin{tabular}{|c|c|c|}
\hline$a^{a^{1}}$ & $a^{2}$ & $d$ \\
\hline $\begin{array}{r}b \\
--\end{array}$ & $\begin{array}{c}1 \\
1 \\
1 \\
i_{1}-\ldots\end{array}$ & $a^{4}$ \\
\hline$a^{6}$ & i & $a^{5}$ \\
\hline
\end{tabular}

Figure 2: Description of binary choice of $f$

\footnotetext{
${ }^{5}$ There is no simpler example with length less than 6 . Note that we do not need (PC2) to rule out a PR-cycle with length 4; P-cycle is a special case of PR-cycle. If we have P-cycle with length of 5, we can reduce the cycle and generate a PR-cycle with length of 4 . But again, this is ruled out by (PE), (PC1) and interchangeability.

${ }^{6}$ If we illustrate them, $\left\{a^{2}\right\}=f\left(\left\{a^{1}, a^{2}, b, a^{3}\right\}\right),\left\{d, a^{4}\right\}=f\left(\left\{a^{2}, d, a^{3}, a^{4}\right\}\right),\left\{a^{3}\right\}=f\left(\left\{b, a^{3}, a^{6}, c\right\}\right)$, $\left\{a^{4}\right\}=f\left(\left\{a^{3}, a^{4}, c, a^{5}\right\}\right),\left\{a^{2}, c\right\}=f\left(\left\{a^{1}, a^{2}, a^{6}, c\right\}\right),\left\{c, a^{5}\right\}=f\left(\left\{a^{2}, d, c, a^{5}\right\}\right), \quad\left\{a^{1}, d, b, a^{4}\right\}=$ $f\left(\left\{a^{1}, d, b, a^{4}\right\}\right)$, and $\left\{b, a^{4}\right\}=f\left(\left\{b, a^{4}, a^{6}, a^{5}\right\}\right)$.
} 
This choice function satisfies (PE), (PC1) and interchangeability. If we assume that the choice function is Nash-rationalizable with a zero-sum game, $(A, \succeq, \preceq)$, the choice function should agree with $\succeq$ over all binary choices. However, $\succeq$ have a $P$-cycle, $a^{1} \succ$ $a^{2} \succ \cdots \succ a^{6} \succ a^{1}$. Thereby the choice function can not be Nash-rationalizable with any P-acyclic relation.

Note that $(\mathrm{PE})$ and $(\mathrm{PC} 1)$ guarantees that individual choices are $P$-acyalic. In a general non-cooperative game, this is enough to guarantee the existence of $P$-acyclic relations $\left(\succeq_{i}\right)_{i \in 1,2}$ which rationalize the choice function. However, if we assume the game is zero-sum, binary choices of one agent directly imply the relation of the other agent. This effect induces a possible $P$-cycle across agents.

It is also restrictive to require observations for all possible subgames when we apply these testable implications. A natural relaxation is to assume that a choice function is defined on $\mathcal{A}^{\prime} \subset \mathcal{A}$. However, Sprumont comments on the difficulties in Nashrationalizability under incomplete observations. Without conditions for rationalizability by general non-cooperative games, it looks even harder to find conditions for rationalizability with zero-sum games.

In classical choice theory, the congruence condition has been studied for incomplete observations (see Richter (1971)). In particular, congruency is defined as the following.

Definition 9 For $\mathcal{B} \subset 2^{X}$ and a choice function $c: \mathcal{B} \longrightarrow 2^{X} \backslash \emptyset$ such that $f(B) \subset B$, define $R^{\prime}$ as,

$$
\text { For all } x, y \in X, x R^{\prime} y \text { if } \exists B \in \mathcal{B} \text { such that }\{x, y\} \subset B \text { and } x \in c(B) \text {. }
$$

If $R^{\prime}$ is consistent, then the choice function is called congruent. ${ }^{7}$

\footnotetext{
${ }^{7}$ For the definition of consistency, see Definition 11.
} 
For individual choice behavior, Richter proves that a choice function is rationalizable by a weak order if and only if it is congruent. However, the following example shows that a naive extension of the congruence condition does not solve the incomplete observation problem in joint behavior.

Example 10 (Individually congruent but not Nash-ratioinalizable) The conceivable game is $2 \times 3$ where agent 1 can choose in $\{U, D\}$ and agent 2 can choose in $\{L, M, R\}$. Three figures show the only three observed games and the choices from them.
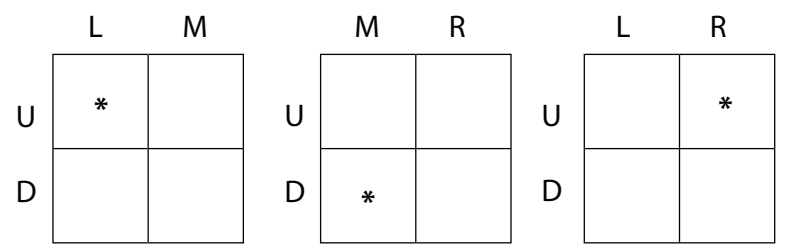

Figure 3: Individually congruent but not Nash-rationalizable

Assuming that the choice function satisfies (PC), we get $\{(U, L)\}=f(\{(U, L),(U, M)\})$ and $\{(U, L)\}=f(\{(U, L),(D, L)\})$ from the first subgame. Applying (PC) to other games, we can verify all $R^{\prime}$ relations in definition 9 for each individual and see the choice functions are congruent. However, assuming that these choices are Nash-rationalizable with $\left(A, \succeq_{1}, \succeq_{2}\right)$, we can derive $(U, R) \succeq_{2}(U, M)$ and $(U, R) \succeq_{1}(D, R)$ from the first and third game. This contradicts the choice from the second game.

This study still leaves out several issues arising from the characteristics of zero-sum games. The first thing to note is that the proof relies on the existence of choice for all $B \in \mathcal{A}$. Considering that we verify the choice is Nash-rationalizable, this implies that all subgames are required to have a pure strategy Nash equilibrium. However, to the best of our knowledge there are no theoretical conditions guaranteeing the existence of pure strategy Nash equilibrium in a finite zero-sum game. Considering the absence of conditions for pure strategy Nash equilibrium, an obvious direction of research would 
investigate either mixed strategies or correlated strategies. However, necessary and sufficient conditions for mixed or correlated strategy rationalizability are also unknown. 


\section{Appendix A Proof of the main theorem}

The necessity of (PE) and (PC) are obvious from the definition of Nash equilibrium. Further, the necessity of interchangeability of choice functions are referred to in Luce and Raiffa (1957) at p.66. In this paper, only the sufficiency of these three conditions is proved by constructing a preference $\succeq$ over $A$ with which for all $B \in \mathscr{A}, f(B)$ coincides with the set of all Nash-equilibria of $(B, \succeq, \preceq)$.

As in example 8, define two relations $\succeq^{*}$ and $\succeq^{* *}$ as follows. For any $a, b \in A$,

$$
\begin{gathered}
a \succeq^{*} b \quad \text { if and only if } \quad a_{2}=b_{2} \text { and } a \in f\left(\left\{a_{1}, b_{1}\right\} \times\left\{a_{2}\right\}\right), \\
a \succeq^{* *} b \quad \text { if and only if } \quad a_{1}=b_{1} \text { and } b \in f\left(\left\{a_{1}\right\} \times\left\{a_{2}, b_{2}\right\}\right)
\end{gathered}
$$

Note that $\succeq^{*}$ and $\succeq^{* *}$ are disjoint. Finally, let $\succeq$ be the union of $\succeq^{*}$ and $\succeq^{* *} .8$ In each line, (PC) is equivalent to Chernoff and Sen's $\beta$, and $\succeq^{*}$ and $\succeq^{* *}$ are defined as analogous with the base-relation. In such case, Sen (1971) showed that $\succeq^{*}$ is a weak order in each column, and $\succeq^{* *}$ is an inverse relation of a weak order in each row; thereby, $\succeq$ is a weak order in both columns and rows. However, $\succeq$ is still incomplete across the lines. Before we proceed with the construction of the weak order over $A$, we need some preliminary definitions.

Definition 11 (Consistent relation) Let $R$ be a relation over $X=\left\{x^{1}, x^{2}, \ldots, x^{l}, \ldots\right\}$ and $P$ be the strict counterpart of $R$. A sequence $x^{1} R x^{2} R \cdots R x^{l} P x^{1}$ is called a PR-cycle (or a cycle). If a relation does not have any cycle, we say that it is consistent.

\footnotetext{
${ }^{8}$ In individual choice theory, given a finite alternative set $X=\{x, y, \ldots\}$ and a choice function $g$, Sen (1971) defines base-relation $\left(R^{*}\right)$ as $x R^{*} y$ if and only if $x \in g(\{x, y\})$

If we arrange player 1's conceivable actions in a column and player 2's actions in a row, thereby constructing a table of joint actions, $\succeq *$ represents the base-relation in each column and $\succeq^{* *}$ represents the base relation in each row in the table, except $\succeq^{* *}$ is defined inversely as compared to the convention.
} 
Definition 12 (Extension) Given any arbitrary binary relation $R$ on $X$, if $R \subset R^{\prime}$ is such that

$$
\begin{aligned}
& x \text { Ry implies } x R^{\prime} y \\
& x P y \text { implies } x P^{\prime} y
\end{aligned}
$$

then $R^{\prime}$ is called an extension of $R$.

In the following, we show that $\succeq$ is consistent, and any weak order extension of $\succeq$ Nash-rationalizes the joint choice function with a zero-sum game.

Lemma $13 \succeq$ is consistent.

Proof of Lemma 13 Suppose by means of contradiction that there exists $\left\{a^{1}, \cdots, a^{N}\right\} \subset$ $A$ such that $a^{1} \succeq a^{2} \succeq \cdots \succeq a^{N} \succ a^{1}$. Since $\succeq$ is the union of two disjoint sets, $\succeq^{*}$ and $\succeq^{* *}$, depending on whether $\left\{a^{i}, a^{j}\right\}$ is in a column or a row, $\succeq$ is either $\succeq^{*}$ or $\succeq^{* *}$.

Without loss of generality, we can restrict attention to cycles of even length, of length at least 4 , whereby the links in the cycle alternate between $\succeq^{* *}$ and $\succeq^{*}$. In order to see that we only need to consider cycles that alternate, note that any cycle containing consecutive $\succeq^{*}$ or $\succeq^{* *}$ can be reduced by means of transitivity to a shorter cycle without consecutive relations. It is also straightforward to check that there are no cycles of length 2 , such as $a^{1} \succeq^{*} a^{2} \succ^{* *} a^{1}$. By definition of $\succeq^{*}, a_{2}^{1}=a_{2}^{2}$ and by definition of $\succeq^{* *}, a_{1}^{1}=a_{1}^{2}$, which together imply that $a^{1}=a^{2}$. However, then we have $a^{1} \succ^{* *} a^{1}$, a contradiction. Finally, we can rule out cycles that are of odd length, since we can shorten any cycle by transitivity to a cycle of even length. For instance, the cycle $a \succeq^{* *} b \succeq^{*} c \succeq^{* *} d \succeq^{*} e \succ^{* *} a$ of length 5 can be reduced to the cycle $b \succeq^{*} c \succeq^{* *} d \succeq^{*} e \succ^{* *} b$ of length 4 .

We demonstrate the case where the cycle begins with $\succeq^{* *}$, however, the case where the cycle begins with $\succeq^{*}$ is analogous. We show by induction that there does not exist a 
cycle of any length.

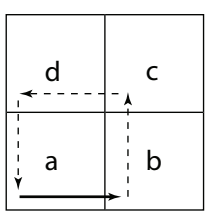

(i)

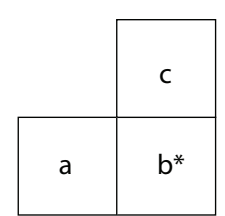

(ii)

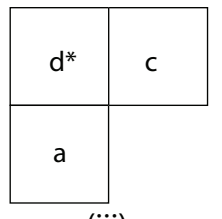

(iii)

Figure 4: A cycle with length of 4

First, we show that there is no cycle with length of 4 . Suppose $a \succeq^{* *} b \succeq^{*} c \succeq^{* *} d \succ^{*}$ $a$. By definition, we have $a_{1}=b_{1}, b_{2}=c_{2}, c_{1}=d_{1}$ and $d_{2}=a_{2}$. Then $\{a, b, c, d\}$ makes a game, depicted in Figure 4. In part (i) of the figure, each dashed arrow corresponds to either $\succeq^{*}$ or $\succeq^{* *}$ and the solid arrow corresponds to $d \succ^{*} a$. The tail of each arrow is the element from the left-hand side of the relation.

Parts (ii) and (iii) of the Figure 4 illustrate the choice function generating $\succeq^{*}$ and $\succeq^{* *}$ for each subgame. Note that $b \in f(\{a, b\}) \cap f(\{b, c\})$, and $d \in f(\{a, d\}) \cap f(\{c, d\}) .{ }^{9}$ Then (PE) implies that $b \in f(\{a, b, c, d\})$ and $d \in f(\{a, b, c, d\})$. Since $f$ is interchangeable, and since $a_{1}=b_{1}$ and $a_{2}=d_{2}, a=\left(b_{1}, d_{2}\right)$ must also be chosen, so that $a \in f(\{a, b, c, d\})$. Likewise, $c=\left(d_{1}, b_{2}\right)$ implies $c \in f(\{a, b, c, d\})$. Finally, (PC) then implies that $a \in$ $f(\{a, d\})$, which contradicts $d \succ^{*} a$. So, there can not be any cycle of length 4 .

Now, we make the induction hypothesis that there is no cycle of length $2(n-1)$ where $n \geq 3$. Given this hypothesis, we show that there is no cycle of length $2 n$.

By reordering the list of individual actions for player 1 and 2 , from a cycle $a^{1} \succeq a^{2} \succeq$ $\cdots a^{2 n} \succ a^{1}$, we can generate the table of joint actions in Figure 3. Here, the dashed arrows and the solid arrow represent the links in the cycle as in Figure 2.

Proof by induction argument requires the steps described below here. Steps 1 to 3 give relations over a subset of $B$ (Figure 10), and step 4 shows another subsets of

\footnotetext{
${ }^{9}$ Note that $\succeq{ }^{* *}$ is defined inversely.
} 


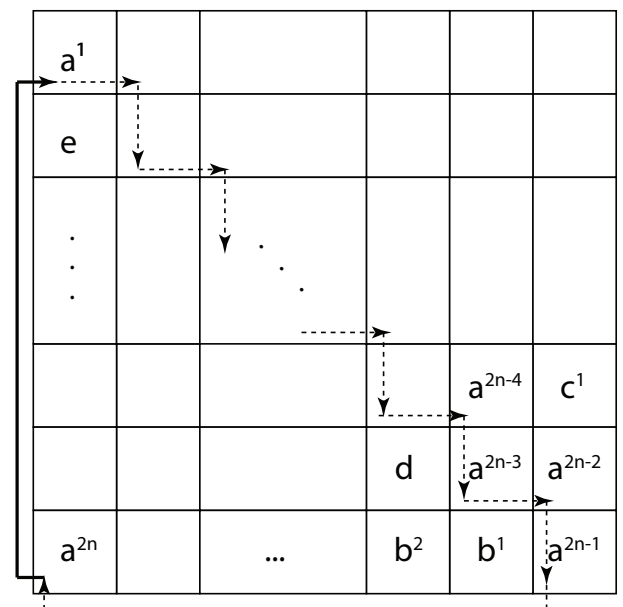

Figure 5: A cycle of length of $2 \mathrm{n}(n \geq 3)$

relations over $B$ (Figure 12-(ii)). Step 5 induces the contradiction of these relations over two subsets.

Step 1: Consider the subgame $\left\{a^{2 n-3}, a^{2 n-2}, a^{2 n-1}, b^{1}\right\}$. In addition to the known relations from the cycle, we can verify $f\left(\left\{a^{2 n-3}, b^{1}\right\}\right)$ and $f\left(\left\{b^{1}, a^{2 n-1}\right)\right\}$. The four cases in Figure 6 below contain all possible cases of $f\left(\left\{a^{2 n-3}, b^{1}\right\}\right)$ and $f\left(\left\{b^{1}, a^{2 n-1}\right)\right\}$. In these two subgames, it must not be the case that either $a^{2 n-3} \in f\left(\left\{a^{2 n-3}, b^{1}\right\}\right)$ and $a^{2 n-1} \in f\left(\left\{b^{1}, a^{2 n-1}\right\}(\right.$ fig (i) $)$, or $b^{1} \in f\left(\left\{a^{2 n-3}, b^{1}\right\}\right)$ and $b^{1} \in f\left(\left\{b^{1}, a^{2 n-1}\right\}\right)$ (fig (ii)).

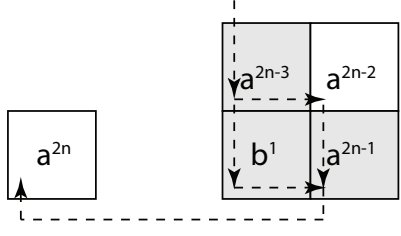

(i.)

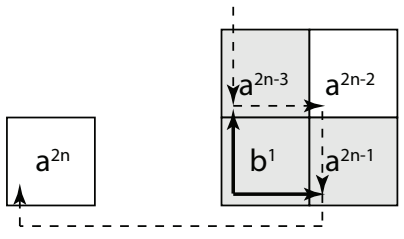

(iii.)

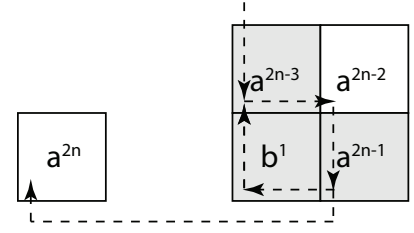

(ii.)

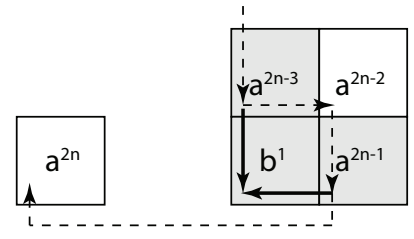

(iv.)

Figure 6: A cycle with length 2n 
In case (i), $a^{2 n-4} \succeq^{*} b^{1}$ and $b^{1} \succeq^{* *} a^{2 n}$ by transitivity of $\succeq^{*}$ in the left column, and by transitivity of $\succeq^{* *}$ in the bottom row. These two relations induce the cycle $a^{1} \succeq \cdots \succeq a^{2 n-4} \succeq b^{1} \succeq a^{2 n} \succ a^{1}$ which is length $2(n-1)$. In case (ii), $b^{1} \in$ $f\left(\left\{a^{2 n-3}, b^{1}\right\}\right) \cap f\left(\left\{b^{1}, a^{2 n-1}\right\}\right)$ and $a^{2 n-2} \in f\left(\left\{a^{2 n-3}, a^{2 n-2}\right\}\right) \cap f\left(\left\{a^{2 n-2}, a^{2 n-1}\right\}\right)$. (PE) induces $a^{2 n-2}$ and $b^{1}$ are in $f\left(\left\{a^{2 n-3}, a^{2 n-2}, a^{2 n-1}, b^{1}\right\}\right)$, and indeed all four joint actions are in $f\left(\left\{a^{2 n-3}, a^{2 n-2}, a^{2 n-1}, b^{1}\right\}\right)$ provided that $f$ is interchangeable. Therefore, we have an indifference relation $\sim$ in $\left\{a^{2 n-3}, b^{1}\right\}$ and $\left\{b^{1}, a^{2 n-1}\right\}$, which gives a special case of (i).

Excluding case (i) and (ii), either (iii) or (iv) is true. We will prove that the induction step is true in case (iii) and omit the case of (iv). The proof that the induction argument is true under case (iv) is omitted here as it can be shown with exactly the same approach as that taken with case (iii).

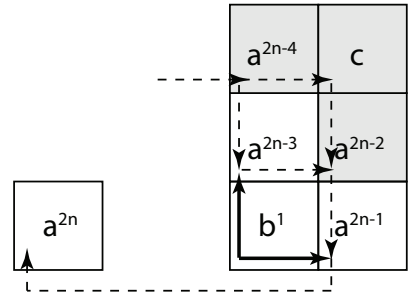

(i.)

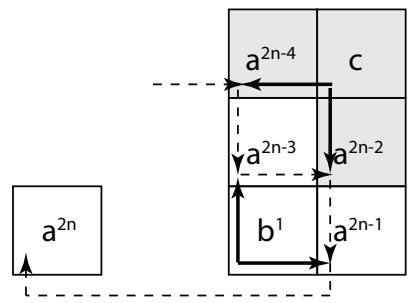

(iii.)

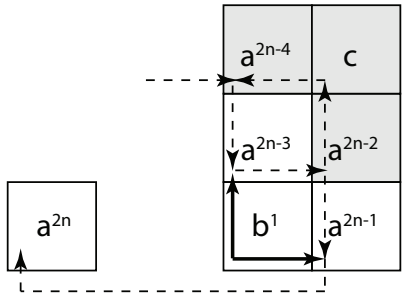

(ii.)

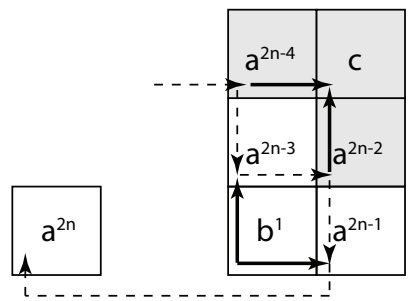

(iv.)

Figure 7: More relations from $f$

Step 2: Figure 7 contains every possible case of $f\left(\left\{a^{2 n-4}, c\right\}\right)$ and $f\left(\left\{c, a^{2 n-2}\right\}\right)$. Using the same argument that used for the case (i), (ii) of $f\left(\left\{a^{2 n-3}, b^{1}\right\}\right)$ and $f\left(\left\{b^{1}, a^{2 n-1}\right\}\right)$, we can rule out the cases of (i) and (ii). In addition, case (iii), $a^{2 n-4}=f\left(\left\{a^{2 n-4}, c\right\}\right)$ and $c=f\left(\left\{c, a^{2 n-2}\right\}\right)$, is not possible, either. This can be shown first by observing 
$b^{1} \succ^{*} a^{2 n-4}$. If it is not the case, completeness of $\succeq^{*}$ in the left column gives $a^{2 n-4} \succeq^{*} b^{1}$, which, combined with $b^{1} \succeq^{* *} a^{2 n}$ by transitivity of $\succeq^{* *}$ in the bottom row, induces the cycle $a^{1} \succeq^{* *} \cdots \succeq^{* *} a^{2 n-4} \succeq^{*} b^{1} \succeq^{* *} a^{2 n} \succ^{*} a^{1}$ whose length is $2(n-1)$.

Once (iii) and $b^{1} \succ^{*} a^{2 n-4}$ are obtained (see Figure 8), consider the set of joint actions $\left\{a^{2 n-4}, c, b^{1}, a^{2 n-1}\right\}$. Any choice from this game violates the (PC) in one subgame of $\left\{a^{2 n-4}, c, b^{1}, a^{2 n-1}\right\}$. Suppose $c \in f\left(\left\{a^{2 n-4}, c, b^{1}, a^{2 n-1}\right\}\right)$, then $c \notin f\left(\left\{a^{2 n-4}, c\right\}\right)$ violates (PC), and likewise any joint action in $\left\{a^{2 n-4}, c, b^{1}, a^{2 n-1}\right\}$ cannot be a choice. Thus the case (iv), $c=f\left(\left\{a^{2 n-4}, c\right\}\right)$ and $a^{2 n-2}=f\left(\left\{c, a^{2 n-2}\right\}\right)$, should be true.

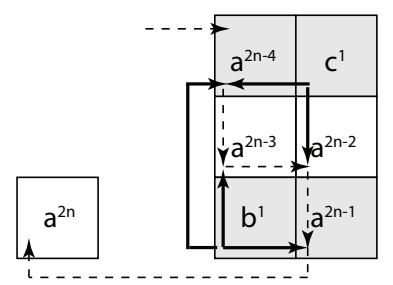

(iii.)

Figure 8: Ruling out the case (iii)

Step 3: Considering $f\left(\left\{a^{2 n-5}, d\right\}\right)$ and $f\left(\left\{d, a^{2 n-3}\right\}\right)$, we can rule out the cases of either $a^{2 n-5} \in f\left(\left\{a^{2 n-5}, d\right\}\right)$ and $a^{2 n-3} \in f\left(\left\{d, a^{2 n-3}\right\}\right)$, or $d \in f\left(\left\{a^{2 n-5}, d\right\}\right)$ and $d \in$ $f\left(\left\{d, a^{2 n-3}\right\}\right)$ by the same argument used for $f\left(\left\{a^{2 n-3}, b^{1}\right\}\right) \& f\left(\left\{b^{1}, a^{2 n-1}\right\}\right)$ and $f\left(\left\{a^{2 n-4}, c\right\}\right)$ $\& f\left(\left\{c, a^{2 n-2}\right\}\right)$ in the previous steps. Accordingly, we only have cases of either $a^{2 n-5}=$ $f\left(\left\{a^{2 n-5}, d\right\}\right)$ and $d=f\left(\left\{d, a^{2 n-3}\right\}\right)$, or $d=f\left(\left\{a^{2 n-5}, d\right\}\right)$ and $a^{2 n-3}=f\left(\left\{d, a^{2 n-3}\right\}\right)$, case (i) or case (ii) in Figure 9, respectively. However, case (i) is ruled out, because once we have $a^{2 n-5} \succ^{*} d$, it must be $a^{2 n-2} \succ^{* *} d$. If not, $d \succeq^{* *} a^{2 n-2}$, then,

1. If the cycle is length $6\left(a^{2 n-5}\right.$ is $a^{1}$ and there is no \# in the fig(i)), $b^{2}$ is equal to $a^{2 n}$. Thus we have $a^{2 n-1} \succeq^{* *} b^{2}$ and $b^{2} \succ^{*} d$ by transitivity of $\succeq^{*}$. As a result, $d \succeq^{* *} a^{2 n-2}$ makes a cycle with length $4, d \succeq^{* *} a^{2 n-2} \succeq^{*} a^{2 n-1} \succeq^{* *} b^{2} \succ^{*} d$, which contradicts the induction hypothesis. 


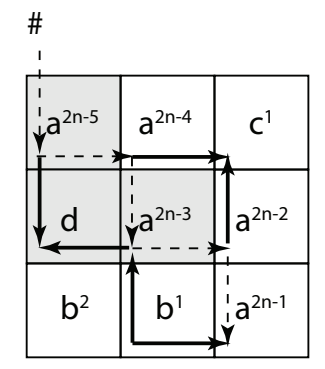

(i.)

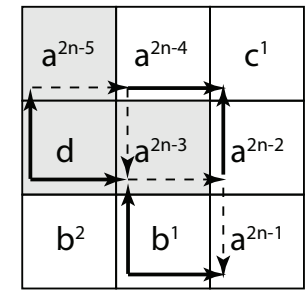

(ii.)

Figure 9: Verifying more relations.

2. If the cycle is length 8 or more (there is $a^{2 n-6}$, '\#' in the fig (i), which is not $\left.a^{1}\right), a^{2 n-6} \succeq^{*} d \succeq^{* *} a^{2 n-2}$ by transitivity of $\succeq^{*}$ and $\succeq^{* *}$ in the left column and the middle row. These relations shorten the cycle, which contradicts the induction hypothesis.

Therefore, in case (i), $a^{2 n-2} \succ^{* *} d$ must be true. However, regardless of what is in $f\left(\left\{a^{2 n-5}, d, c, a^{2 n-2}\right\}\right)$, it violates (PC). For instance, if $d \in f\left(\left\{a^{2 n-5}, d, c, a^{2 n-2}\right\}\right)$ then it must be $d \in f\left(\left\{a^{2 n-5}, d\right\}\right)$, which violates $a^{2 n-5} \succ^{*} d$. Consequently, (ii) in figure 9 must be the case.

By applying steps 2 and 3 sequentially, we can verify more relations. Figure 10 summarizes the result of this process. In the following, step 4 is only for the cycle whose length is greater than or equal to 8 . For a cycle with length of 6 , we already have all the relations that we need to verify in step 4 .

Step 4: Denote the joint action $\left(a_{1}^{2 n-1}, a^{2(n-k)-1}\right)$ as $b^{k}$, the joint action $\left(a_{1}^{2(n-k-1)}, a_{2}^{2(n-k)}\right)$ as $c^{k}$, where $k=1,2, \ldots, n-2$. Figure 10 shows how $b^{i}$ and $c^{i},(1 \leq i \leq n-2)$, are located. Let $\tau$ be a function from $\left\{b^{1}, b^{2}, \ldots, b^{n-2}\right\}$ to $B$ such that, $\tau\left(b^{i}\right)=\left(a_{1}^{2 n-(2 i+1)}, b_{2}^{i}\right)$. Figures 10,11, and 12 show how the function values are located in the game tables. In this step, we show the following claim: 


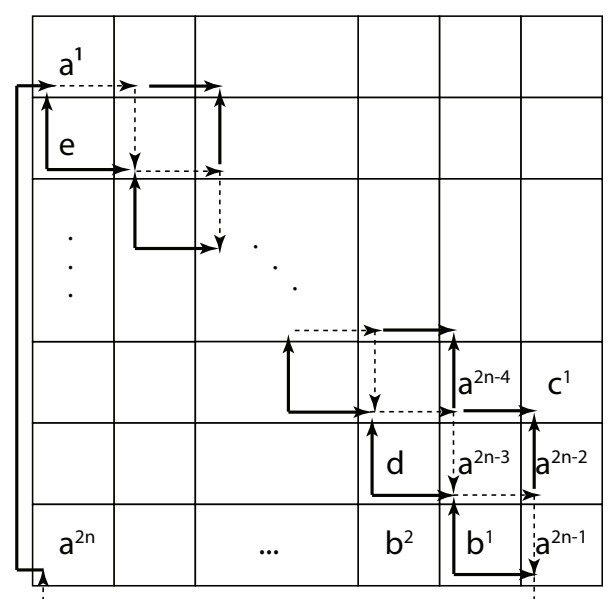

Figure 10: Verified relations by step 2 and 3.

Claim 14 For any $b^{i}(1 \leq i \leq n-2), b^{i} \succ \tau\left(b^{i}\right)$ and $b^{i} \succ a^{2 n-1}$

Proof: We prove this claim by induction. Note that we already assumed that this is true for $b^{1}$ in step 2.

Induction 1: The claim holds for $b^{2}$, that is $b^{2} \succ^{*} \tau\left(b^{2}\right)\left(\right.$ or $\left.a^{2 n-5}\right)$ and $b^{2} \succ^{* *} a^{2 n-1}$.

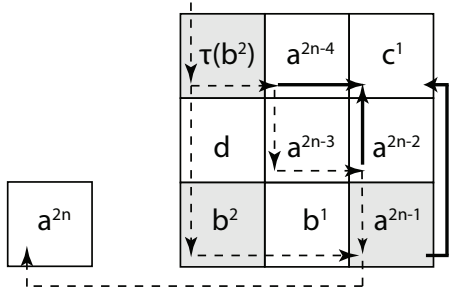

(i.)

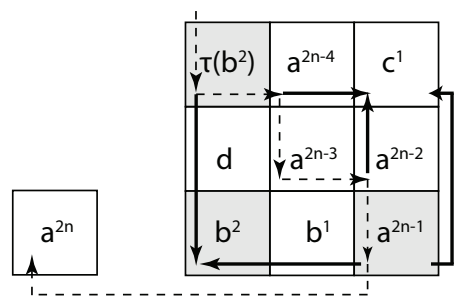

(ii.)

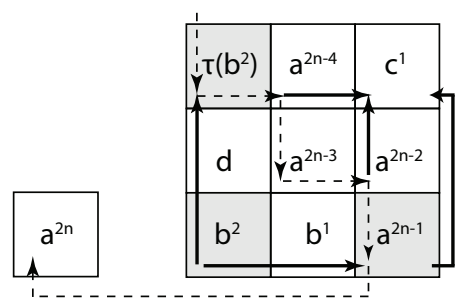

(iii.)

Figure 11: Verifying more relations involving $b^{2}$.

Proof: Considering the subgames, $\left\{\tau\left(b^{2}\right), b^{2}\right\}$ and $\left\{b^{2}, a^{2 n-1}\right\}$ (see Figure 11), it must not be the case that $\tau\left(b^{2}\right) \in f\left(\left\{\tau\left(b^{2}\right), b^{2}\right\}\right)$ and $a^{2 n-1} \in f\left(\left\{b^{2}, a^{2 n-5}\right\}\right)$ (case 
(i)). Otherwise, it shortens the cycle with $a^{2 n-5}=\tau\left(b^{2}\right) \succeq^{*} b^{2} \succeq^{* *} a^{2 n}$ (transitivity in the bottom row is applied.) Therefore, by completeness in each line, we should have either $a^{2 n-1} \succ^{* *} b^{2}$ or $b^{2} \succ^{*} \tau\left(b^{2}\right)$. In the former case, in order not to have a cycle of length 6 , which includes $\left\{\tau\left(b^{2}\right), a^{2 n-4}, a^{2 n-3}, a^{2 n-2}, a^{2 n-1}, b^{2}\right\}, f$ must give $\tau\left(b^{2}\right) \succ^{*} b^{2}$ (fig (ii)) and in the latter case, in order not to have a cycle with length 6 , $f$ must give $b^{2} \succ^{* *} a^{2 n-1}$ (fig (iii)). However, case (ii) is ruled out by considering the subgame, $\left\{\tau\left(b^{2}\right), c^{1}, b^{2}, a^{2 n-1}\right\}$. To show this, observe that $a^{2 n-1} \succ^{*} c^{1}$. Otherwise, $\tau\left(b^{2}\right) \succeq^{* *} c^{1} \succeq^{*} a^{2 n-1}$ shorten the cycle. If the case (ii) is true, then any choice from $\left\{\tau\left(b^{2}\right), c^{1}, b^{2}, a^{2 n-1}\right\}$ violates (PC). For example, if $\tau\left(b^{2}\right) \in f\left(\left\{\tau\left(b^{2}\right), c^{1}, b^{2}, a^{2 n-1}\right\}\right)$, then it must be true that $\tau\left(b^{2}\right) \in f\left(\left\{\tau\left(b^{2}\right), c^{1}\right\}\right)$, which contradicts to $\tau\left(b^{2}\right) \succ^{* *} c^{1}$ (Note again that $\succeq^{* *}$ is defined inversely). Therefore, (iii) must be the case in Figure 11.

Induction 2: If the claim is true for $b^{m-2}$, it is also true for $b^{m}(3 \leq m \leq n-2)$.

Proof: With the same approach as induction 1, $f$ should not give $\tau\left(b^{m}\right) \succeq^{*} b^{m}$ and $b^{m} \succeq^{* *} a^{2 n-1}$, otherwise we have a shorter cycle including $\tau\left(b^{m}\right) \succeq^{*} b^{m} \succeq^{* *} a^{2 n}$. Thus, it must be either $a^{2 n-1} \succ^{* *} b^{m}$ or $b^{m} \succ^{*} \tau\left(b^{m}\right)$. In the former case, not to have a cycle, $b^{m} \succeq^{*} \tau\left(b^{m}\right) \succeq^{* *} \cdots \succeq^{*} a^{2 n-1} \succ^{* *} b^{m}$ which is length $2 m+2 \leq 2(n-1)$, it must be true that $\tau\left(b^{m}\right) \succ^{*} b^{m}$ (case $\left.(\mathrm{i})\right)^{10}$, and in the latter case, not to have the cycle, $\tau\left(b^{m}\right) \succeq^{* *} \cdots \succeq^{*} a^{2 n-1} \succeq^{*} b^{m} \succ^{*} \tau\left(b^{m}\right)$ which is length $2 m+2 \leq 2(n-1)$, it must be true that $b^{m} \succ^{* *} a^{2 n-1}$ (case (ii)). However, case (i) can be ruled out. First, observe that $b^{m-2} \succ^{*} c^{m-1}$ must be true. Otherwise $\tau\left(b^{m}\right) \succeq^{* *} c^{m-1} \succeq^{*} b^{m-2} \succeq^{* *}$ $a^{2 n}$ induces a shorter cycle. In addition, transitivity of $\succeq^{* *}$ in the bottom row gives $b^{m-2} \succ^{* *} b^{m}$. Then, in the subgame, $\left\{\tau\left(b^{m}\right), b^{m}, b^{m-2}, c^{m-1}\right\}$, any choice violates (PC). Therefore, (ii) must be the case in $f\left(\left\{\tau\left(b^{m}, b^{m}\right\}\right)\right.$ and $f\left(\left\{b^{m}, a^{2 n-1}\right\}\right)$.

\footnotetext{
${ }^{10}$ Although we explicitly write the proof only for the case of cycle begins with $\succeq{ }^{* *}$, every single step so far could have been reproduced in cases where cycles begin with $\succ^{*}$, analogously. Here, we used the induction hypothesis, 'there is no cycle of length $2(n-1)$ ', from the counterpart proof of cycles begin with $\succeq *$ and have a strong relation as $\succ^{* *}$
} 
By induction, $b^{i} \succ \tau\left(b^{i}\right)$ and $b^{i} \succ a^{2 n-1}$ for $i=1, \ldots, n-2$, so claim 1 holds.

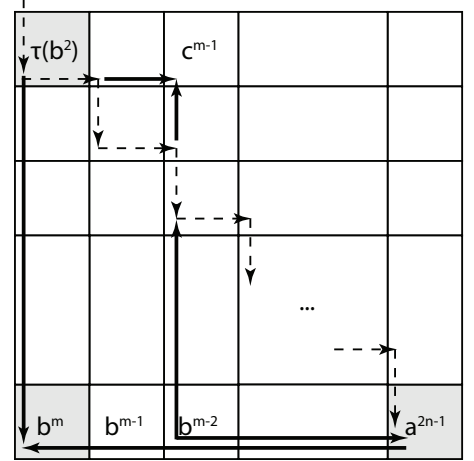

(i.)

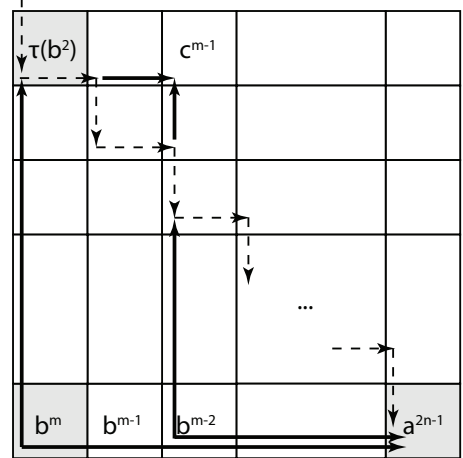

(ii.)

Figure 12: Verifying relations involving $b^{i}$

Step 5: Results from steps 2 and 3 and results from step 4 contradict each other.

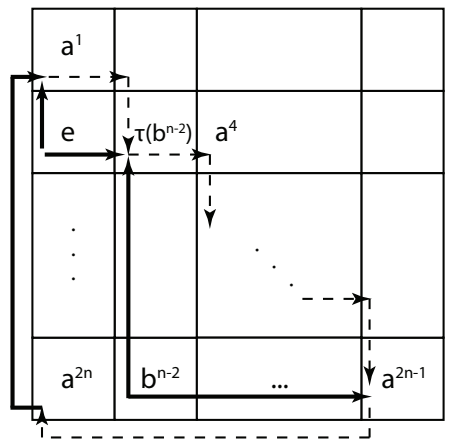

Figure 13: Inducing a contradiction

Proof: If we denote the joint action $\left(\tau\left(b^{n-2}\right)_{1}, a_{2}^{1}\right)$ as $e$ (see Figure 13), then step 2 and 3 gives $e \succ^{*} a^{1}$ and $e \succ^{* *} \tau\left(b^{n-2}\right)$. We showed in step 4 that $b^{n-2} \succ^{*} \tau\left(b^{n-2}\right)$ and $b^{n-2} \succ^{* *} a^{2 n-1}$. Moreover, it must be true that $e \succ^{*} a^{2 n}$, otherwise, $a^{2 n} \succeq^{*} e \succ^{* *} a^{4}$ shortens the cycle. On the other hand, $b^{n-2} \succ^{* *} a^{2 n}$, by transitivity of $\succeq^{* *}$ in the bottom row. Lastly, we can observe that any choice from the subgame, $\left\{e, \tau\left(b^{n-2}\right), a^{2 n}, b^{n-2}\right\}$, violates $(\mathrm{PC})$, and this contradiction completes the proof of step 5, thereby completing the proof of Lemma 13. 


\section{Proof of main theorem}

Claim 15 For all $B \in \mathscr{A}, f(B)$ coincides with the set of all Nash-equilibria of the game $(B, \succeq, \preceq)$.

Proof: Take any $B=B_{1} \times B_{2} \in \mathscr{A}$ and let $\mathrm{NE}(B)$ be the set of all Nash-equilibria of the game $(B, \succeq, \preceq)$. First, to show $f(B) \subset \mathrm{NE}(B)$, take any $b^{*}=\left(b_{1}^{*}, b_{2}^{*}\right) \in f(B)$.

Since $f$ satisfies $(\mathrm{PC}), b^{*} \in f\left(B^{\prime}\right)$ for all $B^{\prime} \in \mathscr{A}$ and $B^{\prime} \subset B$. Therefore, for any $\left\{b^{*},\left(b_{1}, b_{2}^{*}\right)\right\} \subset B, b^{*} \in f\left(\left\{b^{*},\left(b_{1}, b_{2}^{*}\right)\right\}\right)$. By the definition of $\succeq^{*}$, we have $b^{*} \succeq^{*}\left(b_{1}, b_{2}^{*}\right)$, which is equal to $b^{*} \succeq\left(b_{1}, b_{2}^{*}\right)$. Similarly, for any $\left\{b^{*},\left(b_{1}^{*}, b_{2}\right)\right\} \subset B, b^{*} \in f\left(\left\{b^{*},\left(b_{1}^{*}, b_{2}\right)\right\}\right)$. The definition of $\succeq^{* *}$ gives $\left(b_{1}^{*}, b_{2}\right) \succeq^{* *} b^{*}$, which is equal to $\left(b_{1}^{*}, b_{2}\right) \succeq b^{*}$, or $b^{*} \preceq\left(b_{1}^{*}, b_{2}\right)$. Since $b^{*} \succeq\left(b_{1}, b_{2}^{*}\right)$ and $b^{*} \preceq\left(b_{1}^{*}, b_{2}\right)$, for all $\left(b_{1}, b_{2}^{*}\right) \in B$ and $\left(b_{1}^{*}, b_{2}\right) \in B, b^{*}$ is a Nashequilibrium of the game $(B, \succeq, \preceq)$.

Conversely, if $b^{*} \in \mathrm{NE}(B)$, for any $\left(b_{1}, b_{2}^{*}\right) \in B, b^{*} \succeq\left(b_{1}, b_{2}^{*}\right)$. Since, only $\succeq^{*}$ and not $\succeq^{* *}$ is defined in columns, we have $b^{*} \succeq^{*}\left(b_{1}, b_{2}^{*}\right)$ and the definition of $\succeq^{*}$ gives $b^{*} \in f\left(\left\{b^{*},\left(b_{1}, b_{2}^{*}\right)\right\}\right)$. Moreover, $(\mathrm{PE})$ implies $b^{*} \in f\left(B_{1} \times\left\{b_{2}^{*}\right\}\right)(*)$. And $b^{*} \in \mathrm{NE}(B)$ implies $b^{*} \preceq\left(b_{1}^{*}, b_{2}\right)$ for all $\left(b_{1}^{*}, b_{2}\right) \in B$ (or $\left.\left(b_{1}^{*}, b_{2}\right) \succeq b^{*}\right)$. Since, only $\succeq^{* *}$ not $\succeq^{*}$ is defined in rows, we have $\left(b_{1}^{*}, b_{2}\right) \succeq^{* *} b^{*}$. The definition of $\succeq^{* *}$ gives $b^{*} \in f\left(\left\{b^{*},\left(b_{1}^{*}, b_{2}\right)\right\}\right)$ and (PE) induces $b^{*} \in f\left(\left\{b_{1}^{*}\right\} \times B_{2}\right)(* *)$. Lastly, $(*),(* *)$, and $(\mathrm{PE})$ imply that $b^{*} \in f(B)$.

We have shown that $\succeq$ is consistent and $f(B)$ coincides with $\mathrm{NE}(B)$ for all $B \in \mathscr{A}$. Suzumura (1976) shows that a consistent relation has a weak order extension. Observing that the extension generates additional relations only between the joint choices which are not in the same line, this extension does not affect the result of claim 15, so claim 15 is still valid with the weak order extension of $\succeq$. This completes the proof of the main theorem. 


\section{References}

Brown, D. J., And R. L. Matzkin (1996): "Testable Restrictions on the Equilibrium Manifold," Econometrica, 64(6), 1249-1262.

Carvajal, A., I. Ray, and S. Snyder (2004): "Equilibrium behavior in markets and games: testable restrictions and identification," Journal of Mathematical Economics, 40(1-2), 1 - 40, Aggregation, Equilibrium and Observability in honor of Werner Hildenbrand.

Chiappori, P.-A. (1988): "Rational Household Labor Supply," Econometrica, 56(1), $63-90$.

Luce, R. D., AND H. RAIFFA (1957): Games and decisions : introduction and critical survey. Wiley, New York :

Moulin, H. (1985): "Choice functions over a finite set: A summary," Social Choice and Welfare, 2(2), 147-160.

RAY, I., ANd S. K. SNyder (2003): "Observable Implications of Nash and Subgame Perfect Behavior in Extensive Games," SSRN eLibrary.

Ray, I., And L. Zhou (2001): "Game Theory via Revealed Preferences," Games and Economic Behavior, 37(2), 415 - 424.

Richter, M. K. (1971): "Rational Choice," in Preferences, Utility and Demand, John Chipman et al., eds. New York: Harcourt, Brace, Jovanovich.

Samuelson, P. A. (1938): "The Empirical Implications of Utility Analysis," Econometrica, 6(4), 344-356.

SEn, A. K. (1971): "Choice Functions and Revealed Preference," Review of Economic Studies, 38(115), 307-17. 
Sprumont, Y. (2000): "On the Testable Implications of Collective Choice Theories," Journal of Economic Theory, 93(2), 205 - 232.

SuzumurA, K. (1976): "Remarks on the Theory of Collective Choice," Economica, 43(172), 381-390. 